PROCEEDINGS OF THE

AMERICAN MATHEMATICAL SOCIETY

Volume 136, Number 4, April 2008, Pages 1451-1455

S 0002-9939(07)08727-8

Article electronically published on December 28, 2007

\title{
COMPACT METRIZABLE GROUPS ARE ISOMETRY GROUPS OF COMPACT METRIC SPACES
}

\author{
JULIEN MELLERAY \\ (Communicated by Alexander N. Dranishnikov)
}

\begin{abstract}
This note is devoted to proving the following result: given a compact metrizable group $G$, there is a compact metric space $K$ such that $G$ is isomorphic (as a topological group) to the isometry group of $K$.
\end{abstract}

\section{INTRODUCTION}

It is a well-known and useful fact that, if $X$ is a Polish metric space and one endows its isometry group $I s o(X)$ with the topology induced by the product topology on $X^{X}$, then $I s o(X)$ is a Polish group (we recall the definitions of the notions involved in the next section). One may then wonder whether all Polish groups are of that form; this was proved by Gao and Kechris in [2.

Theorem 1.1 (Gao-Kechris). Let $G$ be a Polish group. Then there exists a Polish metric space $Y$ such that $G$ is isomorphic to Iso $(Y)$.

Similarly, it is easy to see that, if $K$ is a compact metric space, then $I s o(K)$ is a compact metrizable group.

Given what we saw above, it is natural to wonder whether the converse holds. The aim of this note is to provide a positive answer to that question.

Theorem 1.2. Let $G$ be a compact metrizable group. Then there exists a compact metric space $K$ such that $G$ is isomorphic to Iso $(K)$.

To obtain the proof, it is natural to try to find a simpler proof of Theorem 1.1 than the one in [2, which is a little buried among other considerations (it is a byproduct of a proof, so no "direct" proof is given).

It turns out that such a simpler proof exists, and it is not very hard to use a variation of it in order to prove Theorem 1.2 .

The paper is organized as follows: first we give a simple proof of Theorem 1.1. then we show how to adapt this proof in order to obtain Theorem 1.2 .

\section{NotATIONS AND DEFINITIONS}

If $(X, d)$ is a complete separable metric space, we say that it is a Polish metric space, and often write it simply as $X$. A Polish group is a topological group whose topology is Polish; if $X$ is a separable metric space, then we denote its isometry

Received by the editors January 10, 2006 and, in revised form, March 7, 2006.

2000 Mathematics Subject Classification. Primary 54H11; Secondary 22A05, 51F99.

(C)2007 American Mathematical Society 
group by $I s o(X)$, and endow it with the pointwise convergence topology, which turns it into a second countable topological group, and into a Polish group if $X$ is Polish (see [1] for a thorough introduction to the theory of Polish groups).

Let $(X, d)$ be a metric space; we say that $f: X \rightarrow \mathbb{R}$ is a Katětov map iff

$$
\forall x, y \in X|f(x)-f(y)| \leq d(x, y) \leq f(x)+f(y) .
$$

These maps correspond to one-point metric extensions of $X$. We denote by $E(X)$ the set of all Katětov maps on $X$; we endow it with the sup-metric, which turns it into a complete metric space.

If $f \in E(X)$ and $S \subseteq X$ are such that $f(x)=\inf (f(s)+d(x, s): s \in S)$, we say that $S$ is a support of $f$, or that $S$ controls $f$.

It is useful here to note the following easy fact: if $f, g \in E(X)$ are both supported by some set $S$, then $d(f, g)=\sup _{s \in S}|f(s)-g(s)|$.

Note that $X$ isometrically embeds in $E(X)$ via the Kuratowski embedding $x \mapsto$ $\delta_{x}$, where $\delta_{x}(y)=d(x, y)$, and that one has, for any $f \in E(X)$, that $d\left(f, \delta_{x}\right)=f(x)$.

\section{Proofs of the theOREMS}

As promised, we begin by proving Theorem 1.1 .

Let $G$ be a Polish group, and let $d$ be a left-invariant distance on $G$. Let $X$ be the completion of $(G, d)$. Then the left translation action of $G$ on itself extends to an action by isometries of $G$ on $X$, and it is not hard to check that this provides an isomorphic embedding of topological groups of $G$ in $I s o(X)$. We identify $G$ with the corresponding (closed) subgroup of $I s o(X)$, and make the additional assumption that $X$ is of diameter $\leq 1$.

Claim. For all $\varphi \in I s o(X) \backslash G$, there exist $x_{1}, \ldots, x_{m} \in X$ and $\varepsilon>0$ such that $2 m \varepsilon=\min \left(d\left(x_{l}, x_{k}\right)\right)$, and

$$
V_{\varphi}=\left\{\psi \in I s o(X): \forall k=1, \ldots, m d\left(\psi\left(x_{k}\right), \varphi\left(x_{k}\right)\right)<\varepsilon\right\} \subseteq I s o(X) \backslash G .
$$

Proof. Obvious.

Choose for all $\varphi \in I s o(X) \backslash G$ such a $V_{\varphi}$; then, since $I s o(X) \backslash G$ is Lindelöf, there are $\left\{\varphi_{i}\right\}_{i \geq 1}$ such that $I s o(X) \backslash G=\bigcup_{i \geq 1} V_{\varphi_{i}}$. We let $V_{\varphi_{i}}=\{\psi \in I s o(X): \forall k=$ $\left.1, \ldots, m_{i} d\left(y_{k}^{i}, \psi\left(x_{k}^{i}\right)\right)<\varepsilon_{i}\right\}$.

For each $i \geq 1$ we define maps $f_{i}, g_{i} \in E(X)$ :

$$
\begin{aligned}
& f_{i}(x)=\min _{1 \leq k \leq m_{i}}\left(1+d\left(x, x_{k}^{i}\right)+2(k-1) \varepsilon_{i}\right), \\
& g_{i}(x)=\min _{1 \leq k \leq m_{i}}\left(1+d\left(x, y_{k}^{i}\right)+2(k-1) \varepsilon_{i}\right) .
\end{aligned}
$$

If $\varphi \in I s o(X)$, we let $\varphi^{*}$ denote its (unique) extension to $E(X)$, defined by $\varphi^{*}(f)(x)=f\left(\varphi^{-1}(x)\right)$.

The introduction of the maps $f_{i}, g_{i}$ is justified by the following lemma.

Lemma 3.1. $\forall \varphi \in I s o(X) \forall i \geq 1\left(\varphi \in V_{\varphi_{i}}\right) \Leftrightarrow\left(d\left(\varphi^{*}\left(f_{i}\right), g_{i}\right)<\varepsilon_{i}\right)$.

Proof of Lemma 3.1, Let $\varphi \in V_{\varphi_{i}}$. Then $\varphi^{*}\left(f_{i}\right)\left(y_{k}^{i}\right)=\min _{1 \leq k \leq m_{i}}\left(1+d\left(y_{k}^{i}, \varphi\left(x_{k}^{i}\right)\right)\right.$ $\left.+2(k-1) \varepsilon_{i}\right)$, so the various inequalities involved imply that $\varphi^{*}\left(f_{i}\right)\left(y_{k}^{i}\right)=1+$ $d\left(\varphi\left(x_{k}^{i}\right), y_{k}^{i}\right)+2(k-1) \varepsilon_{i}$, hence $\left|\varphi^{*}\left(f_{i}\right)\left(y_{k}^{i}\right)-g_{i}\left(y_{k}^{i}\right)\right|<\varepsilon_{i}$. Similarly, $g_{i}\left(\varphi\left(x_{k}^{i}\right)\right)=$ $1+d\left(\varphi\left(x_{k}^{i}\right), y_{k}^{i}\right)+2(k-1) \varepsilon_{i}$, so $\left|\varphi^{*}\left(f_{i}\right)\left(\varphi\left(x_{k}^{i}\right)\right)-g_{i}\left(\varphi\left(x_{k}^{i}\right)\right)\right|<\varepsilon_{i}$. Since $\varphi^{*}\left(f_{i}\right)$ and $g_{i}$ are both supported by the set $\left\{\varphi\left(x_{k}^{i}\right)\right\}_{k=1, \ldots, m_{i}} \cup\left\{y_{k}^{i}\right\}_{k=1, \ldots, m_{i}}$, the inequalities above are enough to show that $d\left(\varphi^{*}\left(f_{i}\right), g_{i}\right)<\varepsilon_{i}$. 
Conversely, let $\varphi \in I s o(X)$ be such that $d\left(\varphi^{*}\left(f_{i}\right), g_{i}\right)<\varepsilon_{i}$. We prove by induction on $k=1, \ldots, m$ that $d\left(\varphi\left(x_{k}^{i}\right), y_{k}^{i}\right)<\varepsilon_{i}$.

To see that this is true for $k=1$, remark that we have that $g_{i}\left(\varphi\left(x_{1}^{i}\right)\right)<1+\varepsilon_{i}$, so that we must have $g_{i}\left(\varphi\left(x_{1}^{i}\right)\right)=g_{i}\left(y_{1}^{i}\right)+d\left(y_{1}^{i}, \varphi\left(x_{1}^{i}\right)\right)$. In turn, this implies that $d\left(y_{1}^{i}, \varphi\left(x_{1}^{i}\right)\right)<\varepsilon_{i}$.

Suppose now that we have proved the result up to rank $k-1 \leq m-1$.

Note that we have this time that $\left.g_{i}\left(\varphi\left(x_{k}^{i}\right)\right)<1+\varepsilon_{i}+(2 k-1) \varepsilon_{i}{ }^{*}\right)$. Also, we know that for all $l<k$ we have $d\left(\varphi\left(x_{k}^{i}\right), \varphi\left(x_{l}^{i}\right)\right)>2 m_{i} \varepsilon_{i}$, and $d\left(\varphi\left(x_{l}^{i}\right), y_{l}^{i}\right)<\varepsilon_{i}$. Thus, $d\left(\varphi\left(x_{k}^{i}\right), y_{l}^{i}\right)>\left(2 m_{i}-1\right) \varepsilon_{i}$ for all $l<k$.

It is then clear that $\left(^{*}\right)$ implies that $g_{i}\left(\varphi\left(x_{k}^{i}\right)\right)=g_{i}\left(y_{k}^{i}\right)+d\left(y_{k}^{i}, \varphi\left(x_{k}^{i}\right)\right)$, which means that $d\left(y_{k}^{i}, \varphi\left(x_{k}^{i}\right)\right)<\varepsilon_{i}$.

We now let $F_{0}=X, F_{i}=\overline{\left\{\varphi^{*}\left(f_{i}\right): \varphi \in G\right\}} \subset E(X)$, and $Z=\overline{U F_{i}}$. Note that $Z$ is a Polish metric space, since it is closed in $E(X)$, which is complete, and it admits a separable dense subset.

Also, it is important to remark that Lemma 3.1 implies that $d\left(\psi^{*}\left(f_{i}\right), g_{i}\right) \geq \varepsilon_{i}$ for all $\psi \in G$; in other words, $d\left(F_{i}, g_{i}\right) \geq \varepsilon_{i}$ for all $i$.

Lemma 3.2. Any element $\varphi$ of $G$ extends (uniquely) to an isometry $\varphi^{Z}$ of $Z$, and

$$
\left\{\varphi^{Z}: \varphi \in G\right\}=\left\{\varphi \in I s o(Z): \forall i \geq 0 \varphi\left(F_{i}\right)=F_{i}\right\}
$$

Proof of Lemma 3.2, The first assertion is easy to prove: since $\varphi^{*}\left(F_{i}\right)=F_{i}$ for all $\varphi \in G$, we see that $\varphi^{*}(Z)=Z$ for all $\varphi \in G$. The fact that this extension is unique is a classical consequence of the definition of the distance on $E(X)$; see for instance [3].

It is also clear that $\left\{\varphi^{Z}: \varphi \in G\right\} \subseteq\left\{\varphi \in I s o(Z): \forall i \geq 0 \varphi\left(F_{i}\right)=F_{i}\right\}$; to prove the converse, take $\varphi \in I s o(Z)$ such that $\varphi(X)=X$ and $\varphi\left(F_{i}\right)=F_{i}$ for all $i$.

Then $\varphi_{\left.\right|_{X}}$ is an isometry of $X$ such that $d\left(\left(\varphi_{\left.\right|_{X}}\right)^{*}\left(f_{i}\right), g_{i}\right)=d\left(\varphi\left(f_{i}\right), g_{i}\right) \geq \varepsilon_{i}$ for all $i$, which means that $\varphi_{\left.\right|_{X}} \notin V_{\varphi_{i}}$ for all $i$, so that $\varphi_{\left.\right|_{X}} \in G$ and we are done.

To complete the proof of Theorem 1.1, we replace the distance $d$ on $Z$ by $\frac{d}{1+d}$; then Lemma 3.2 is still true, and $\operatorname{diam}(\mathrm{Z}) \leq 1$.

We conclude as in [2]: for all $i \in \mathbb{N}$ choose a point $y_{i}$ and assume that all these points are distinct and not in $Z$. We define a distance $d$ on $Y=Z \cup\left\{y_{i}\right\}$ extending the distance on $Z$ by $d\left(y_{i}, z\right)=(i+2)+d\left(z, F_{i}\right)$ for $z \in Z$; $d\left(y_{i}, y_{j}\right)=$ $\inf _{z \in Z}\left(d\left(y_{i}, z\right)+d\left(y_{j}, z\right)\right)$ if $i \neq j$.

Then $(Y, d)$ is Polish, and we claim that $G$ is isomorphic to $I s o(Y)$.

Indeed, any element $\varphi$ of $G$ has a unique isometric extension $\varphi^{Y}$ to $Y$, and the mapping $\varphi \mapsto \varphi^{Y}$ is continuous. Conversely, let $\psi$ be an isometry of $Y$; necessarily $\psi(Z)=Z$ (a point $y$ is in $Z$ iff there exists $y^{\prime} \in Y$ such that $0<d\left(y, y^{\prime}\right) \leq 1$ ), therefore $\psi\left(y_{i}\right)=y_{i}$ for all $i$. Since $F_{i}=\left\{z \in Z: d\left(z, y_{i}\right)=i+2\right\}$, we must have $\psi\left(F_{i}\right)=F_{i}$ for all $i$. So, we see that there is some $\varphi \in G$ such that $\psi_{\left.\right|_{Z}}=\varphi^{Z}$, so that $\psi=\varphi^{Y}$.

To conclude the proof of Theorem 1.1, recall that any bijective, continuous morphism between two Polish groups is actually bicontinuous; therefore the map $\varphi \mapsto \varphi^{Z}$ is an isomorphism from $G$ onto $I s o(Z)$.

Now we show how to adapt this proof in order to prove Theorem 1.2 .

We may again assume that $G$ has more than two elements. Let $d$ be an invariant metric on $G$; the metric space $X=(G, d)$ is compact, and $G$ embeds topologically 
in $I s o(X)$, via the mapping $g \mapsto(x \mapsto g \cdot x)$. We again identify $G$ with the corresponding (closed) subgroup of $I s o(X)$, and make the additional assumption that $X$ is of diameter $\leq 1$. We again choose, for all $\varphi \in I s o(X) \backslash G$, a $V_{\varphi}$ as in the claim; there are $\left\{\varphi_{i}\right\}_{i \geq 1}$ such that $I s o(X) \backslash G=\bigcup_{i \geq 1} V_{\varphi_{i}}$.

We again denote $V_{\varphi_{i}}=\left\{\psi \in I s o(X): \forall 1 \leq k \leq m_{i} d\left(y_{k}^{i}, \psi\left(x_{k}^{i}\right)\right)<\varepsilon_{i}\right\}$.

For each $i \geq 1$ we define slightly different maps $f_{i}, g_{i}$ :

$$
\begin{aligned}
& f_{i}(x)=\min \left(\min _{1 \leq k \leq m_{i}}\left(1+\frac{1}{2^{i}}+d\left(x, x_{k}^{i}\right)+2(k-1) \varepsilon_{i}\right), 1+\frac{1}{2^{i}}+2 m_{i} \varepsilon_{i}\right), \text { and } \\
& g_{i}(x)=\min \left(\min _{1 \leq k \leq m_{i}}\left(1+\frac{1}{2^{i}}+d\left(x, y_{k}^{i}\right)+2(k-1) \varepsilon_{i}\right), 1+\frac{1}{2^{i}}+2 m_{i} \varepsilon_{i}\right) .
\end{aligned}
$$

If $\varphi \in I \operatorname{so}(X)$, we let $\varphi^{*}$ denote its (unique) extension to $E(X)$; we have again that

$$
\forall \varphi \in I s o(X) \forall i \geq 1\left(\varphi \in V_{\varphi_{i}}\right) \Leftrightarrow\left(d\left(\varphi^{*}\left(f_{i}\right), g_{i}\right)<\varepsilon_{i}\right) .
$$

Now, we let $Y$ be the set of $f \in E(X)$ such that

$$
\exists n \geq 1 \exists x_{1}, \ldots, x_{n} \quad \forall x f(x)=\min \left(\min _{1 \leq i \leq n}\left(1+2(i-1) \varepsilon+d\left(x, x_{i}\right)\right), 1+2 n \varepsilon\right),
$$

where $2 n \varepsilon=\min \left(d\left(x_{i}, x_{j}\right)\right)$. (For $n=1$ one gets $g$ defined by $g(x)=1$ for all $x \in X$.)

Lemma 3.3. $Y$ is compact.

Proof. Let $\left(f_{i}\right)$ be a sequence of maps in $Y$, and let $x_{1}^{i}, \ldots, x_{n_{i}}^{i}$ be points witnessing the fact that $f_{i} \in Y$.

Then, either we can extract a sequence $f_{\varphi(i)}$ such that $n_{\varphi(i)} \rightarrow+\infty$, or $\left(n_{i}\right)$ is bounded.

In the first case, note that, since $X$ is totally bounded, one must necessarily have that $\min _{1 \leq j<k \leq n_{\varphi(i)}} d\left(x_{j}^{\varphi(i)}, x_{k}^{\varphi(i)}\right) \rightarrow 0$ when $n \rightarrow+\infty$, so that the definition of $f_{i}$ ensures that $f_{\varphi(i)} \rightarrow g$. $i$.

In the other case, we may extract a subsequence $f_{\psi(i)}$ such that $n_{\psi(i)}=n$ for all

We assume that $\min _{1 \leq j<k \leq n} d\left(x_{j}^{\psi(i)}, x_{k}^{\psi(i)}\right) \geq \delta$ for some $\delta>0$ (if not, we can conclude as in the first case that some subsequence of $\left(f_{\psi(i)}\right)$ converges to $\left.g\right)$. But then, up to another extraction, we can suppose that $x_{1}^{\psi(i)} \rightarrow x_{1}, \ldots, x_{n}^{\psi(i)} \rightarrow x_{n}$. This implies that

$$
\min _{1 \leq j<k \leq n} d\left(x_{j}^{\psi(i)}, x_{k}^{\psi(i)}\right) \rightarrow \min _{1 \leq j<k \leq n} d\left(x_{j}, x_{k}\right),
$$

and one checks easily that $f_{\psi(i)} \rightarrow f$ for some $f \in Y$.

We let, for all $i \geq 1, F_{i}=\left\{\varphi^{*}\left(f_{i}\right): \varphi \in G\right\}$ and $Z=X \cup Y \cup \cup F_{i}$. We claim that $Z$ is compact; to see that, it is enough to show that any sequence $\left(z_{n}\right)$ of elements of $\bigcup F_{i}$ admits a subsequence converging to some $z \in Z$.

We know by definition that $z_{n}=\varphi_{n}\left(f_{i_{n}}\right)$ for some $\varphi_{n} \in G$ and $i_{n} \in \mathbb{N}$. Since $G$ is compact, we may assume that $\varphi_{n} \rightarrow \varphi$, so that it is enough to show that $\left(f_{i_{n}}\right)$ admits a subsequence converging to some $z^{\prime} \in Z$.

We may of course assume that $i_{n} \rightarrow+\infty$. Note that, by definition, $f_{i_{n}}=\frac{1}{2^{i_{n}}}+h_{n}$, for some $h_{n} \in Y$; since $Y$ is compact, and $i_{n} \rightarrow+\infty$, we are done.

The end of the proof is very similar to that of Theorem 1.1, only a bit simpler (that is why we have chosen the $f_{i}$ 's more carefully this time): we pick $k \in E(Z)$ such that $d(k, z)=2 \operatorname{diam}(Z)+d(z, X)$, and let $\{k\} \cup Z=K$. 
$K$ is compact, any element of $G$ extends uniquely to an isometry of $K$, and the extension morphism is continuous.

So, we only need to prove that all isometries of $K$ are extensions of elements of $G$; to that end, let $\psi \in I s o(K)$.

We see that $\psi(k)=k$, so $\psi(Z)=Z$.

Also, since $X=\{z \in Z: d(z, k)=2 \operatorname{diam}(Z)\}$, we must have $\psi(X)=X$. Similarly, one has $F_{i}=\left\{z \in Z: d(z, X)=1+\frac{1}{2^{i}}\right\}$, so $\psi\left(F_{i}\right)=F_{i}$.

We may now conclude as above: $\psi_{\left.\right|_{X}} \notin V_{\varphi_{i}}$ for all $i$, so $\psi_{\left.\right|_{X}} \in G$, and we are done.

\section{Closing Remark}

The proof above is a "coding" proof, which means that, given a compact group $G$, we manage to encode a compact metric space $K$ in such a way that its isometry group is isometric to $G$. To do this, we forget all additional structures on $G$; a consequence is that the space $K$ constructed is bad even if the group $G$ was nice. For instance, assume that $G$ is a compact Lie group, endowed with a biinvariant metric. Then $I s o(G)$ is naturally endowed with a structure of compact Riemannian manifold, and is strictly bigger than $G$ in general, since for instance if $G$ is nonabelian both left and right multiplication are isometries. Even if $G$ is abelian, $I \operatorname{so}(G)$ does not need to be equal to $G$ (for instance, $I s o\left(S^{1}\right)$ is isomorphic to $S^{1} \times\{-1,1\}$, where $S^{1}$ is the group of complex numbers of modulus 1 ).

So, the following question arises: is every compact Lie group the isometry group of some compact Riemannian manifold? To the best of the author's knowledge, this is an open question; one may think of several similar questions (in general, one may wonder, given some class of metric spaces, what is precisely the class of groups obtained as isometry groups of spaces in this class. Results similar to Theorems 1.1 and 1.2 would be nice illustrations of the fact that groups are geometric objects.

\section{ACKNowledgments}

I would like to thank Alekos Kechris, who told me about the problem studied here; I am also grateful to the anonymous referee.

\section{REFERENCES}

1. H.Becker and A.S Kechris, The Descriptive Set Theory of Polish Group Actions, London Math. Soc. Lecture Notes Series, 232, Cambridge University Press (1996). MR1425877 (98d:54068)

2. S. Gao and A.S Kechris, On the classification of Polish metric spaces up to isometry, Memoirs of Amer. Math. Soc., 766, Amer. Math. Soc. (2003). MR1950332 (2004b:03067)

3. M. Katětov, On universal metric spaces, Proc. of the 6th Prague Topological Symposium (1986), Frolik (ed). Helderman Verlag, Berlin, pp. 323-330 (1988). MR0952617 (89k:54066)

Université Paris 6, Boîte 186, 4 Place Jussieu, Paris Cedex 05, France

E-mail address: melleray@math.jussieu.fr 